\title{
A Rare Presentation of Cecal Diverticulitis in a Patient With Cystic Fibrosis
}

\author{
Manrique Guerrero ${ }^{a}$, Mariam Selevany ${ }^{b}, \mathrm{~d}$, Monica Sridhar ${ }^{\mathrm{c}}$, \\ Paul Hanna ${ }^{\mathrm{a}}$, Jamshed Zuberia
}

\begin{abstract}
Cecal diverticulitis (CD) is a rarely seen and studied condition in American medicine. $\mathrm{CD}$ presents in a manner that is almost clinically indistinguishable from acute appendicitis. Both CD and acute appendicitis present with exceedingly similar symptoms including, but not limited to, right lower quadrant (RLQ) pain, fever, and vomiting. The diagnosis of CD is usually made during an operation and is seldom caught preoperatively. Although a fair number of CD cases have been reported since its discovery, only a handful of patients concurrently had a history of cystic fibrosis (CF). We report a rare case of CD that was initially believed to be appendicitis in a male patient with $\mathrm{CF}$. Intraoperatively, a diagnosis of $\mathrm{CD}$ was reached due to identification of a single diverticulum in the cecal region with inflammatory changes consistent with microperforation. A partial cecectomy was performed and the patient was discharged 4 days later with resolution of symptoms.
\end{abstract}

Keywords: Cecal diverticulitis; Cystic fibrosis; Diverticulitis; Appendicitis; Cecum

\section{Introduction}

A diverticulum or a sac-like protrusion of the colonic wall can become inflamed, leading to a condition known as acute diverticulitis. A vast majority of acute diverticulitis cases in industrialized nations are left-sided, mainly involving the sigmoid colon [1]. Although rare, diverticulitis of the right colon, including the cecum, may occur and typically presents with the right lower quadrant (RLQ) pain usually associated with acute appendicitis [2,3]. Diverticulitis of any location is frequently mild when pericolic fat and mesentery wall off a small perforation. However, larger perforations and more extensive disease

Manuscript submitted January 16, 2019, accepted February 11, 2019

aSt. Joseph's University Medical Center, Paterson, NJ, USA

'Rowan SOM, Stratford, NJ, USA

'Robert Wood Johnson Medical School, New Brunswick, NJ, USA

${ }^{\mathrm{d}}$ Corresponding Author: Manrique Guerrero, St. Joseph's University Medical

Center, Paterson, NJ 07503, USA. Email: manrique_guerrero@yahoo.com

doi: https://doi.org/10.14740/jcs374 lead to abscess formation, intestinal rupture, and diffuse peritonitis. The etiology of left-sided diverticular disease is usually due to long standing constipation secondary to a low-fiber diet, mostly seen in Western nations. However, right-sided diverticular disease is usually due to a congenital abnormality and, according to several studies, is more common in younger Asian men [4-6].

Cystic fibrosis (CF) is a genetic, ethnically specific disease with a frequency of roughly 1 in 2,500 births in the USA [7]. CF consists of exocrine gland dysfunction due to a gene mutation; this gland dysfunction leads to multi-organ involvement, including, but not limited to the pancreas, the lungs, the liver, and the bowel [8]. CF may manifest in adolescents as bowel pathology resulting in bowel obstruction. Recognized causes of bowel obstruction in CF patients include meconium ileus, intussusception, distal intestinal obstruction syndrome, and postoperative adhesions $[9,10]$. An increased incidence of $14 \%$ between appendiceal diverticulosis and CF has been reported as opposed to $1 \%$ in the general population $[10,11]$. However, cecal diverticulitis (CD) and CF have not been noted to have any specific relationship. This case report describes a rare incidence of $\mathrm{CD}$ in a young male patient with a history of CF.

\section{Case Report}

A 22-year-old Caucasian man with a history of CF presented to the emergency department with acute abdominal pain 2 days prior to presentation. The patient described the pain as sharp, intermittent, and located in the RLQ. A history of fever, nausea, vomiting, diarrhea, anorexia, or dysuria was not endorsed. However, the patient did endorse prior multiple occurrences of constipation. Home medications included respiratory treatments and pancreatic enzymes. Laboratory studies done in the emergency department revealed a leukocytosis of $13.8 \mathrm{k} / \mathrm{mm}^{3}$, neutrophil shift within normal limits, and no bands present on the complete blood count (CBC). Abdominal computed tomography (CT) demonstrated a mild amount of fluid in the right paracolic gutter adjacent to the cecum. The appendix appeared to be normal (Fig. 1).

An increase in stool burden was also noted (Fig. 2). On physical exam, the patient elicited RLQ tenderness to palpation. The patient was admitted to the surgical floor for serial abdominal exams. He was kept nothing by mouth (NPO) and was given intravenous fluids only. Overnight and into the next 


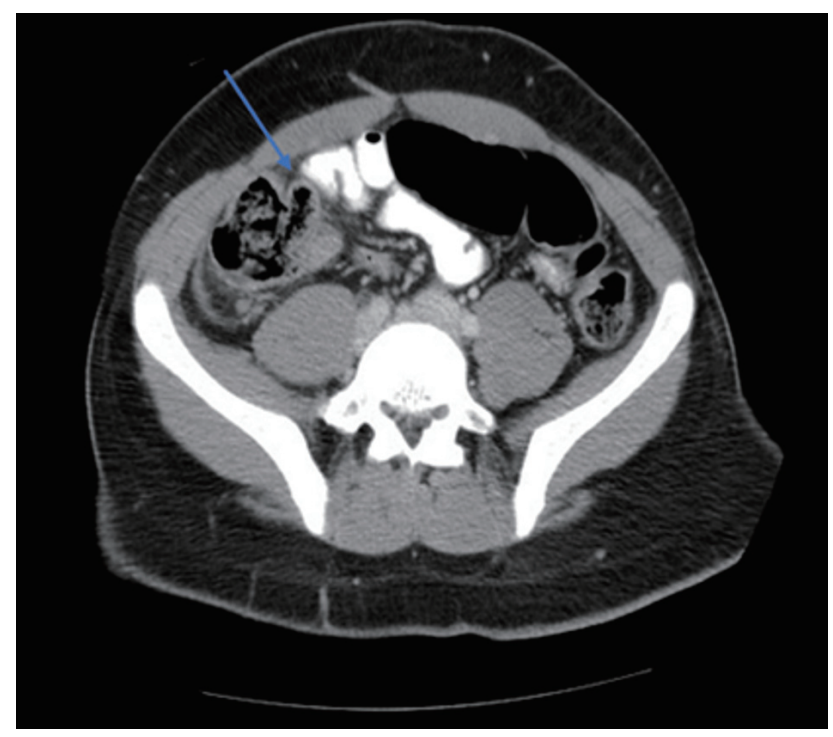

Figure 1. Abdominal CT, an axial view, demonstrated a mild amount of fluid in the right paracolic gutter adjacent to the cecum. A colonic extension (blue arrow).

morning, the patient continued to complain of RLQ pain with increased tenderness only in the RLQ. Additionally, the patient's neutrophil count increased to $17 \mathrm{k} / \mathrm{mm}^{3}$. He was assumed to have acute appendicitis and was taken to the operating room (OR) for an open appendectomy. Intraoperatively, the appendix was found to be within normal limits. Surprisingly, the patient was found to have a solitary diverticulum in the cecum with surrounding inflammatory changes and microperforation. Based on pathology, a diagnosis of CD was reached. A partial cecectomy along with an appendectomy was performed. Postoperatively, the patient continued to improve clinically; however, constipation despite cessation of opiates was noted. In addition to the CD, the patient was diagnosed with distal intestinal obstruction syndrome and given Golytely. On the fifth postoperative day, the patient was clinically improved and was discharged home on a strict bowel regimen.

\section{Discussion}

CF is usually caused as a result of a dysfunctional CF transmembrane regulator (CFTR) gene which carries the function of salt excretion [12]. CF can cause gastrointestinal manifestations due to viscous secretions and dysmotility. Additionally, distal intestinal obstruction syndrome secondary to CF can be caused by inspissated intestinal secretions; these obstructions commonly occur at the ileocecal location [9]. A stark difference in the presentation of our patient as opposed to other patients in the literature was that he complained of 5 days of constipation prior to arrival in the emergency room. Both CD and appendicitis rarely present with a complaint of constipation. However, based on clinical presentation and imaging, the constipation was found to be an incidental finding secondary to distal intestinal obstruction syndrome.

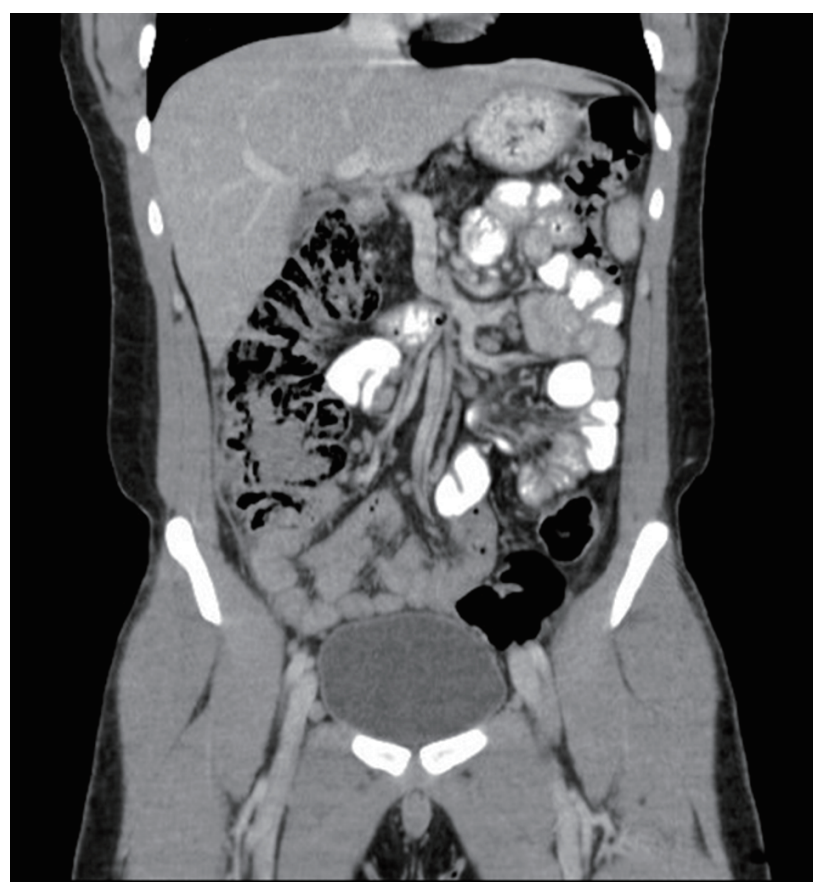

Figure 2. Abdominal CT, a coronal view, showed inspissated stool in ascending colon.

Although appendicitis may be a relatively common finding in a pediatric patient, it is said to be fairly uncommon and difficult to diagnose in a patient with CF [13]. In CF patients, the appendix is frequently swollen or enlarged due to inspissated eosinophilic secretions; however, the occurrence of appendicitis in patients with CF is $1 \%$ as opposed to $7 \%$ in the general population possibly secondary to the protective effect of mucus secretions [14]. For this very reason, in patients with a history of $\mathrm{CF}$, the clinician should expand the differential diagnosis when a patient presents with the pathognomonic signs of appendicitis. Both diseases, CD and acute appendicitis, tend to present with RLQ pain, fever, tenderness, and nausea. Minor differences include decreased incidence of nausea and vomiting in patients that have CD or rightsided diverticulitis as opposed to appendicitis [15]. As seen in our patient, right-sided diverticulitis mostly begins with prolonged vague pain in the RLQ region as opposed to pain starting in the epigastric region that migrates over to the RLQ [2]. Our patient never endorsed initial epigastric pain, which can be a clinical clue pointing towards a diagnosis of $C D$ as opposed to appendicitis. According to 306 case reports taken from literature, the average age of patients that present with $\mathrm{CD}$ is 40 years old, with men comprising roughly $60 \%$ of the total [16]. Our patient was much younger than the average presenting age of $\mathrm{CD}$; however, of the one other reported case of a patient that had CF and CD concurrently, the age of presentation tended to be towards the younger side [11]. Therefore, when considering the differentials of abdominal pain in a patient with $\mathrm{CF}, \mathrm{CD}$ should be included regardless of age at the time of presentation. Also, as seen in our patient, the majority of patients with CD have symptoms for a duration of $48 \mathrm{~h}$ or less before presenting for treatment. However, 
it should be noted that a substantial number of patients have a long prodromal phase of RLQ pain before seeking treatment [16].

It has been hypothesized that inspissated secretions in patients with CF fill the appendix and provide a protective effect against inflammation, thereby reducing the incidence of appendicitis $[11,14]$. However, while the incidence of appendicitis is reduced, the incidence of diverticulitis, specifically appendiceal diverticulitis, is increased [11]. Hypertrophy of the muscular wall alongside with distension of the appendix due to inspissated secretions leads to diverticula formation at weak points in the wall, mostly where the muscle is penetrated by vessels. In a way that is similar to appendiceal diverticular formation, the pathogeneses of cecal diverticular formation in CF patients could possibly be secondary to increased intraluminal pressure by inspissated secretions filling the cecum. Although the incidence of CD concurrently with a diagnosis of $\mathrm{CF}$ is basically negligible in current literature, it is important to consider a diagnosis of CD in order to save the patient from unnecessary surgery [17].

\section{Conclusions}

This case report describes a 22-year-old Caucasian man with a past medical history of CF who presented with symptoms initially assumed to be appendicitis but was found to be CD intraoperatively based on pathology. Enough cases of CD have been described in the literature in order to provide some generalizations regarding this disease. CD has been noted to occur more commonly in Asian/Eastern populations, has been noted to have an average age of presentation of 40 years old, and is commonly confused with appendicitis. However, when considering a patient with $\mathrm{CF}$, these generalizations no longer apply. A vast majority of patients afflicted with $\mathrm{CF}$ are of Caucasian descent so the chances that the patient will be of Asian descent are highly unlikely [18]. Additionally, CD should be expected to occur in a $\mathrm{CF}$ patient at a much younger age than the average $\mathrm{CD}$ patient. Finally, when a patient presents with "typical appendicitis signs", remember that appendicitis has a decreased incidence in CF patients and consider broadening your differential.

\section{Acknowledgments}

None.

\section{Funding}

This research did not receive any specific grant from funding agencies in the public, commercial, or not-for-profit sectors.

\section{Conflict of Interest}

None.

\section{Informed Consent}

None to declare.

\section{Author Contributions}

MG participated in the case and patient care, and wrote the case report. M Selevany, M Sridhar, PH, and JZ edited the case report.

\section{References}

1. Parks TG. Natural history of diverticular disease of the colon. Clin Gastroenterol. 1975;4(1):53-69.

2. Fischer MG, Farkas AM. Diverticulitis of the cecum and ascending colon. Dis Colon Rectum. 1984;27(7):454458.

3. Markham NI, Li AK. Diverticulitis of the right colon experience from Hong Kong. Gut. 1992;33(4):547-549.

4. Lo CY, Chu KW. Acute diverticulitis of the right colon. Am J Surg. 1996;171(2):244-246.

5. Lee IK, Jung SE, Gorden DL, Lee YS, Jung DY, Oh ST, $\mathrm{Kim} \mathrm{JG}$, et al. The diagnostic criteria for right colonic diverticulitis: prospective evaluation of 100 patients. Int J Colorectal Dis. 2008;23(12):1151-1157.

6. Lee IK, Lee YS, Kim SJ, Gorden DL, Won DY, Kim HJ, Cho HM, et al. Laparoscopic and open surgery for right colonic diverticulitis. Am Surg. 2010;76(5):486-491.

7. Ratjen F, Doring G. Cystic fibrosis. Lancet. 2003;361(9358):681-689.

8. De Boeck K, Wilschanski M, Castellani C, Taylor C, Cuppens H, Dodge J, Sinaasappel M, et al. Cystic fibrosis: terminology and diagnostic algorithms. Thorax. 2006;61(7):627-635.

9. Colombo C, Ellemunter H, Houwen R, Munck A, Taylor C, Wilschanski M, Ecfs. Guidelines for the diagnosis and management of distal intestinal obstruction syndrome in cystic fibrosis patients. J Cyst Fibros. 2011;10(Suppl 2):S24-28.

10. Benya EC, Nussbaum-Blask AR, Selby DM. Colonic diverticulitis causing partial bowel obstruction in a child with cystic fibrosis. Pediatr Radiol. 1997;27(12):918919.

11. George DH. Diverticulosis of the vermiform appendix in patients with cystic fibrosis. Hum Pathol. 1987;18(1):7579.

12. Sabharwal S. Gastrointestinal manifestations of cystic fibrosis. Gastroenterol Hepatol (N Y). 2016;12(1):43-47.

13. Shields MD, Levison H, Reisman JJ, Durie PR, Canny GJ. Appendicitis in cystic fibrosis. Arch Dis Child. 1991;66(3):307-310.

14. McCarthy VP, Mischler EH, Hubbard VS, Chernick MS, di Sant'Agnese PA. Appendiceal abscess in cystic fibrosis. A diagnostic challenge. Gastroenterology. 1984;86(3):564-568.

15. Case TC, Shea CE, Jr. Acute diverticulitis of the cecum. 
Am J Surg. 1953;85(2):134-141.

16. Graham SM, Ballantyne GH. Cecal diverticulitis. A review of the American experience. Dis Colon Rectum. 1987;30(10):821-826.

17. Park HC, Lee BH. Suspected uncomplicated cecal diverticulitis diagnosed by imaging: initial antibiotics vs laparoscopic treatment. World J Gastroenterol. 2010;16(38):4854-4857.

18. Accurso FJ. Update in cystic fibrosis 2005. Am J Respir Crit Care Med. 2006;173(9):944-947. 